\title{
Modelling and shape optimization of an actuator
}

\author{
Charles-Henri Bruneau • Frédéric Chantalat • \\ Angelo Iollo • Bastien Jordi · Iraj Mortazavi
}

Received: 21 December 2012 / Revised: 2 May 2013 / Accepted: 9 May 2013 / Published online: 14 June 2013

(C) Springer-Verlag Berlin Heidelberg 2013

\begin{abstract}
The aim of this work is to optimize an actuator design so that the flow profile at its exit section is as close as possible to a target profile. The method is founded on the penalization and level-set methods to solve direct and inverse problems on Cartesian meshes The optimization process is written and applied both for Stokes and Navier-Stokes flows. The results show that the method can be successfully applied to the non linear problem to improve the flow profile of an actuator even if the target cannot be totally reached.
\end{abstract}

Keywords MEMS · Shape optimization · Navier-Stokes flow

\section{Introduction}

Reducing or at least controlling the consumption of energy has become one of the main challenge of our societies. Among human activities transportation is one of the most energy consuming. Many researches have been performed these last years to reduce the drag of vehicles. Among them active flow control around bluff bodies such as planes, cars

C.-H. Bruneau $(\bowtie) \cdot$ F. Chantalat · A. Iollo $\cdot$ B. Jordi ·

I. Mortazavi

Univ. Bordeaux, IMB, UMR 5251, 33400 Talence, France

e-mail: bruneau@math.u-bordeaux1.fr

C.-H. Bruneau · F. Chantalat · A. Iollo · B. Jordi - I. Mortazavi

CNRS, IMB, UMR 5251, 33400 Talence, France

C.-H. Bruneau · F. Chantalat · A. Iollo - B. Jordi - I. Mortazavi INRIA, Team MC2, 33400 Talence, France or trucks is largely widespread. Active flow control needs actuators that modify a flow by providing an electronically controllable disturbance. Some of these actuators are named micro-electro-mechanical systems (MEMS). They control the flow through micro jets that interact with the flow in the boundary layer and either modify the vortex shedding or change the vortices behaviour. They have characteristic lengths of the order of the milimeter, that combine electrical and mechanical components and that are fabricated using integrated circuit batch-processing technologies (Gad-el-Hak 1999). In the car industry these actuators are settled side by side to form as much as possible a continuous line. Then, they can generate a global drag reduction effect, modifying the vortex dynamics in some critical locations of the body.

The present investigation is motivated in this framework and concerns the shape optimization (Céa1986, Gad-el-Hak 1999 of an actuator so that the flow at the exit section is almost constant. It corresponds to a constrained optimal shape design (Osher and Santosa 2001) using a fastmarching level-set approach (Sethian 2001). Considering an initial obstacle embedded in a two-dimensional Stokes flow, the goal is to find a geometry that has the same area as the initial one and that minimizes a given objective function. Hence, starting from an actuator with four channels at the entrance section, the aim is to modify the shape of the actuator to get the required profile at the exit section.

Many methods have been derived to solve such problems these last decades as can be seen in (Bendsøe and Sigmund 2003; Mohammadi and Pironneau 2010) and references therein. Among all these methods in topology optimization it is important to quote the solid isotropic material with penalization (SIMP) approach (Bendsøe and Sigmund 2003; Brackett et al. 2010) and the coupling of level-set method 
with topological derivation (Allaire and Jouve 2006; Duan et al. 2008; Osher and Santosa 2001). Besides there are many applications to shape optimization for fluids (Amstutz 2005; Borrvall and Petersson 2003; Gersborg-Hansen et al. 2005; Guillaume and Sid 2004; Mohammadi and Pironneau 2010; Zhou and Li 2008).

The method used here for this shape optimization is described in (Chantalat et al. 2009) and (Chantalat 2009). For the sake of completeness the main lines are recalled in this paper to ease the reading. Therefore the first section is devoted to the modelling and the numerical approximation of the penalized Navier-Stokes equations and some details on the used level-set method. Then the shape optimization is discussed for a Stokes flow and very precise results are presented. Finally the method is extended to the nonlinear case thanks to the computation of the unstable steady solution.

\section{Modelling and numerical simulation}

The actuator is made of four channels converging to an exit section. The challenge is to get at this exit section the required velocity profile. In order to settle the optimization process we add to the actuator domain a channel whose width is equal to the width of the exit section of the actuator (see Fig. 1). A part of that channel called $\Omega_{\text {strip }}$ is used to evaluate the cost function of the minimization problem. The flow enters the computational domain through the four entrance sections of the actuator and goes out through an exit section beyond the strip as shown in the figure. The position and the size of the four entrance sections as well as the position and the size of the exit section of the actuator are fixed and cannot be changed. This is a considerable constraint for the optimization process. In facts, if the actuator could be changed to a single channel to get a Poiseuille flow this study would have no interest.

\subsection{Modelling}

The two-dimensional computational domain $\Omega=\Omega_{F} \cup$ $\Omega_{S} \cup \Gamma$ includes both fluid and solid domains, $\Gamma$ is the interface between the fluid and the solid domains. It is a fixed rectangular domain on which an uniform Cartesian mesh is defined. To handle the solid parts, the penalized Navier-Stokes equations are used. It consists in adding a penalization term $U / K$ to the momentum equation. So the non dimensional penalized Navier-Stokes equations for an incompressible fluid read:

$$
\left\{\begin{array}{l}
\partial_{t} U+(U . \nabla) U-\frac{1}{R e} \Delta U+\nabla p+\frac{U}{K}=0 \text { in } \Omega \times[0, T] \\
\nabla . U=0 \text { in } \Omega \times[0, T]
\end{array}\right.
$$

where $U=\left(u_{1}, u_{2}\right)$, and $p$ are the flow velocity and the pressure and the simulation is performed for $t \in[0, T]$. $K$ is the non dimensional permeability coefficient used to represent the solid parts (see (Angot et al. 1999) and (Bruneau and Mortazavi 2008) for more details), in practice this coefficient is set to $10^{-8}$ in the solid domain and to $10^{16}$ in the fluid domain to recover the genuine NavierStokes equations. The non-dimensional Reynolds number $R e$ is computed using the mean velocity at the entrance sections and the width of the exit section.

The unsteady (1) are associated to the initial datum and boundary conditions:

$$
\left\{\begin{array}{l}
U(x, 0)=0 \text { in } \Omega \\
U=(1,0) \text { on }\left(S_{\text {ent }_{1}} \cup S_{\text {ent }_{2}} \cup S_{\text {ent }_{3}} \cup S_{\text {ent }_{4}}\right) \times[0, T] \\
U=(0,0) \text { on }\left(\partial \Omega \cap \partial \Omega_{S}\right) \times[0, T] \\
\sigma(U, p) n+\frac{1}{2}(U \cdot n)^{-}\left(U-U_{\text {ref }}\right)=\sigma\left(U_{\text {ref }}, p_{\text {ref }}\right) n \text { on } \\
\quad S_{\text {exit }} \times[0, T]
\end{array}\right.
$$

Fig. 1 Computational domain. Left: The fluid domain is denoted $\Omega_{F}$ and the solid domain $\Omega_{S}$. The vertical line corresponds to the exit section of the actuator. Right: The four entrance sections of the actuator are numbered on the left hand side. The strip on which the cost function will be evaluated starts at the exit section of the actuator. $S_{\text {exit }}$ denotes the exit section of the fluid computational domain
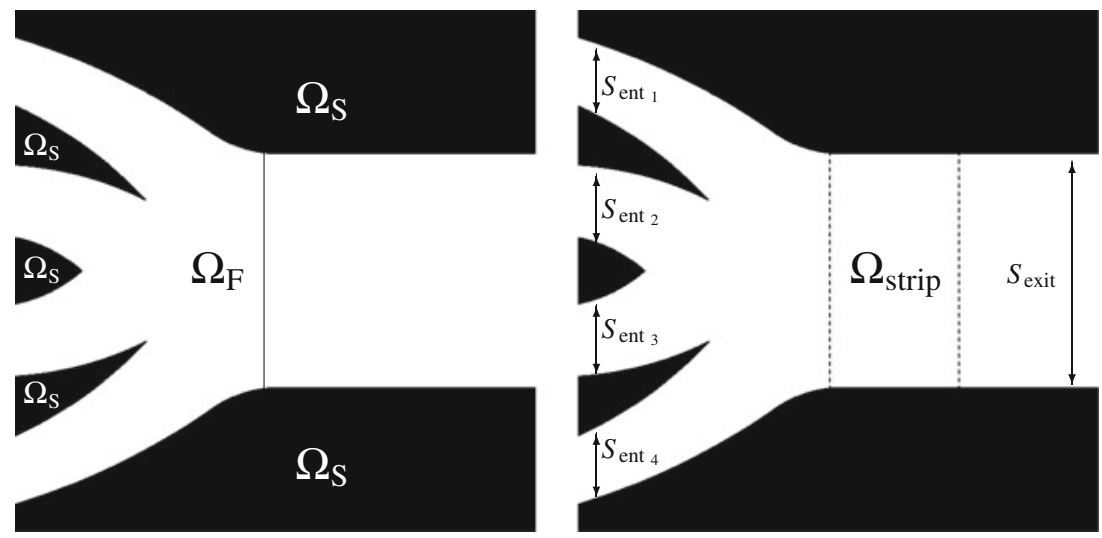
where $\sigma(U, p)=1 / \operatorname{Re}\left(\nabla U+\nabla U^{t}\right)-p I$ is the stress tensor, $n$ is the unit normal pointing outside of the domain and for a real number $a$ the notation $a=a^{+}-a^{-}$is used. The reference flow $\left(U_{\text {ref }}, p_{\text {ref }}\right)$ is taken equal to the computed flow obtained just before the exit section at each iteration. The no-slip boundary condition on the interface $\Gamma$ is enforced by the penalization method as the velocity will be the same order than $K$

\subsection{Level-set method}

The main feature of the level-set method is to be able to represent a curve or a surface without an explicit parametrization (Osher and Fedkiw 2001; Osher and Sethian 1988). Indeed, the fluid-solid interface $\Gamma$ is considered as the zero level-set of a function $\phi$, defined as follows:

$\phi(x, t)=\left\{\begin{array}{l}\phi(x, t)=+d \text { if } \quad x \in \Omega_{S}(t), \\ \phi(x, t)=-d \text { if } x \in \Omega_{F}(t), \\ \phi(x, t)=0 \text { if } x \in \Gamma(t),\end{array}\right.$

where $d$ is the distance from $x$ to the interface $\Gamma$.

Let $V$ be a continuous velocity vector field such that $\frac{d x}{d t}=V(x, t)$ for $x \in \Gamma$. If the displacement is determined by this vector field $V$, the evolution of the level-set function $\phi$ satisfies a first-order transport equation. Introducing a normal velocity $V_{n}=V \cdot \frac{\nabla \phi}{|\nabla \phi|}$ it can be formulated as an Hamilton-Jacobi problem:

$\left\{\begin{array}{l}\frac{\partial \phi}{\partial t}+V_{n}|\nabla \phi|=0 \\ \phi(x, 0)=\phi_{0}\end{array}\right.$

where $\phi_{0}$ gives the initial position of $\Gamma$. A uniform Cartesian mesh twice as fine as the mesh used for solving problem (1) is used to track the motion of the interface. The gradients of all quantities can be approximated using a finite difference discretization and the norm of the level-set gradient is:

$$
\begin{aligned}
|\nabla \phi|^{2}= & \left(\frac{\phi_{i, j}-\min \left(\phi_{i+1, j}, \phi_{i-1, j}\right)}{\Delta x_{1}}\right)^{2} \\
& +\left(\frac{\phi_{i, j}-\min \left(\phi_{i, j+1}, \phi_{i, j-1}\right)}{\Delta x_{2}}\right)^{2},
\end{aligned}
$$

where $\Delta x_{1}$ and $\Delta x_{2}$ stand for the space discretization steps and $\phi_{i, j}$ is the value of $\phi$ at point $\left(i \Delta x_{1}, j \Delta x_{2}\right.$ ). This technique is extensively described in (Allaire and Jouve 2006; Chantalat et al. 2009; Mohammadi and Pironneau 2010; Osher and Sethian 1988). The discretization is performed using the high-order WENO5 scheme (Jiang and Shu 1996).
A spacing procedure of the function $\phi$ has to be periodically performed in a neighbourhood of the interface. This procedure is handled by the fast-marching method (Sethian 1996).

\subsection{Numerical method and results of direct problem}

The system of (1), (2) is solved by a strongly coupled approach for the physical unknowns $(U, p)$. The numerical simulation does not use any turbulence model. The time discretization in (1) is achieved using a second-order Gear scheme with explicit treatment of the convection term. That means that $\partial_{t} U$ is approximated at time $n \delta t$ by $\left(3 U^{n}-4 U^{n-1}+U^{n-2}\right) /(2 \delta t)$ and that the nonlinear convection term is computed on the right hand side in the form $2\left(U^{n-1} \cdot \nabla\right) U^{n-1}-\left(U^{n-2} \cdot \nabla\right) U^{n-2}$ to get second order. All the linear terms are treated implicitly on the left hand side at time $n \delta t$ and discretized via a second-order centered finite differences scheme. The CFL condition related to the convection term requires a time step $\delta t$ of the order of magnitude of the space step for the non dimensional problem, which is relevant to have a good accuracy of the evolution and does not induce too much cpu time consumption. A third-order finite differences upwind scheme is used for the space discretization of the convection terms (Bruneau and Saad 2006). The efficiency of the resolution is obtained by a multigrid procedure using a cell-by-cell relaxation smoother.

The results are presented on a $640 \times 640$ cells uniform mesh that has been chosen after a detailed grid convergence study in order to achieve accurate computations (Bruneau and Saad 2006). This mesh is kept for all the computations performed in this paper. The Reynolds number $R e=4669$ corresponds to the required velocity of the actuator $\bar{V}=$ $30 \mathrm{~m} / \mathrm{s}$. In Fig. 2 are plotted an instantaneous vorticity field and the mean vorticity field. The first one clearly shows that Karman alleys develop at the sharp edges of the solid parts. So the mean velocity at the exit section of the actuator shows significant variations of amplitude across the section, as can be seen in Fig. 3. The aim of this work is to optimize the design of the actuator to have a better velocity profile.

\section{Shape optimization for a Stokes flow}

In this section, as a first step in the investigation, the shape optimization method detailed in (Chantalat et al. 2009) is applied to Stokes flow in order to estimate the performance of the procedure. Inside the domain presented in Fig. 1, a viscous incompressible flow satisfying the Stokes equations is considered. In that case the expected velocity profile at the exit section is close to Poiseuille flow. The goal of this 

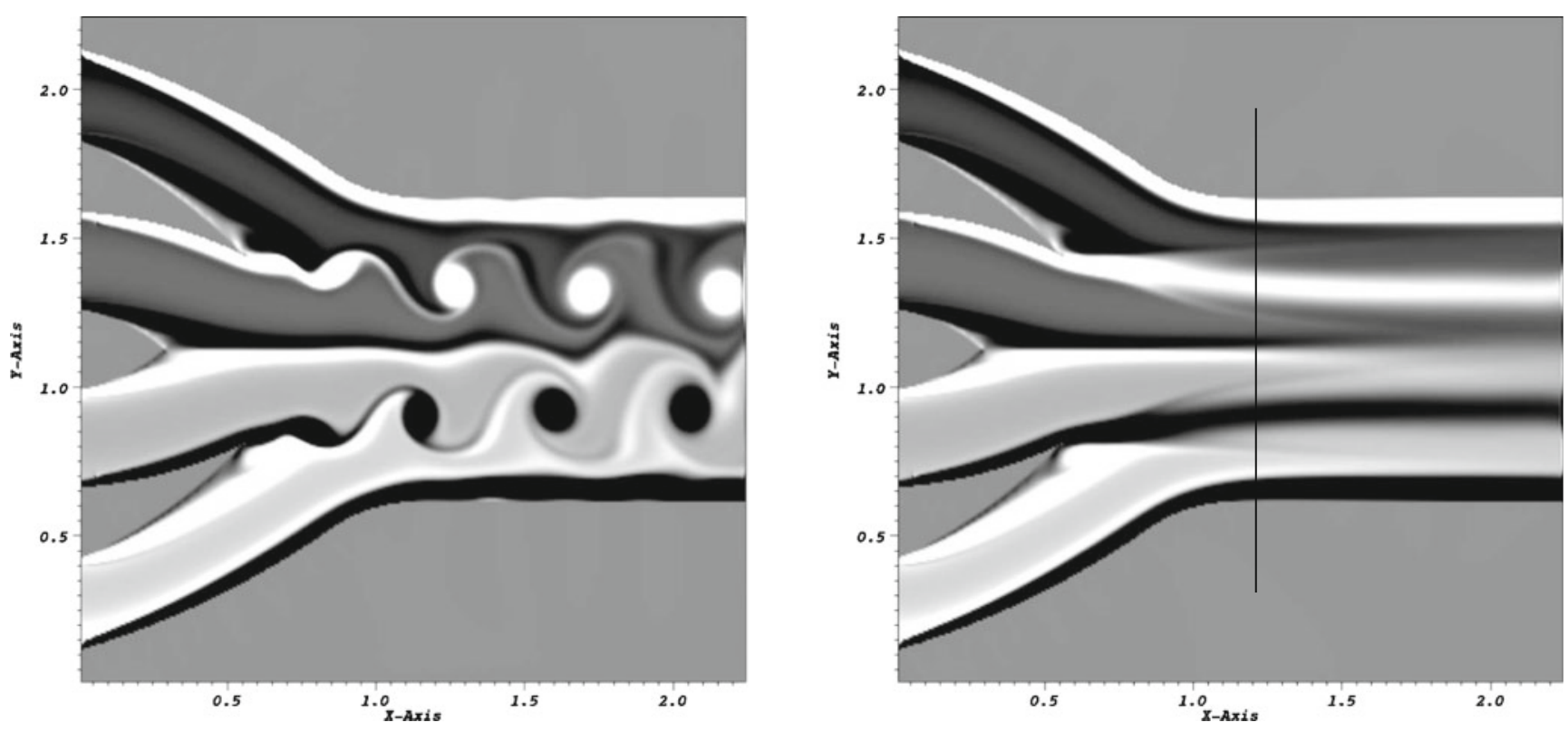

Fig. 2 Instantaneous vorticity field on the left and mean vorticity field on the right in the whole computational domain. The vertical line corresponds to the exit section of the actuator

section is to modify the geometry of the actuator to minimize the deviation between the computed velocity $U$ and the target profile $U_{\text {target }}$ that is equal to Poiseuille profile.

\subsection{Direct problem}

The Stokes flow is described by the steady Stokes equations in $\Omega_{F}$. As in the previous section, the penalization method (Angot et al. 1999) is used to handle the solid parts on the whole computational domain $\Omega$. So the equations read:

$\left\{\begin{array}{l}-\frac{1}{R e} \Delta U+\nabla p+\frac{U}{K}=0 \text { in } \Omega \\ \nabla \cdot U=0 \text { in } \Omega\end{array}\right.$

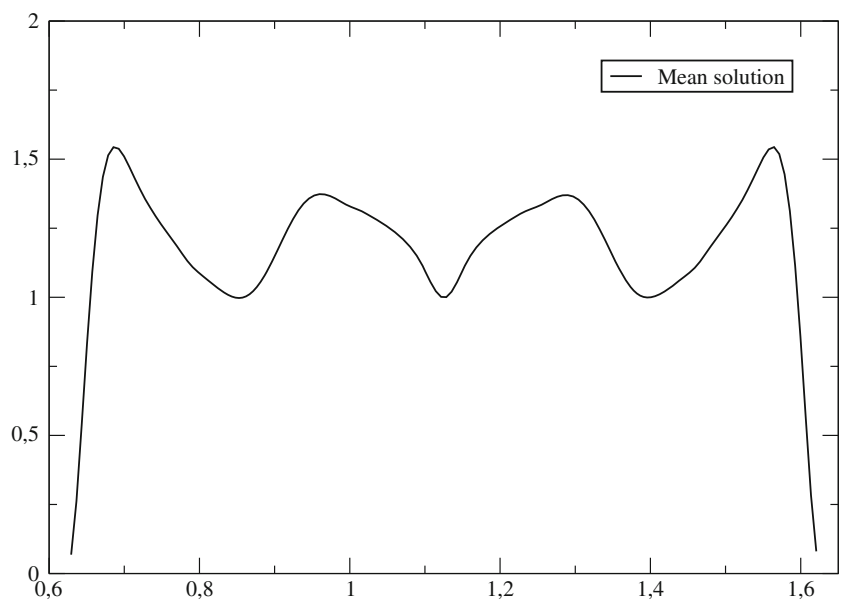

Fig. 3 Profile of the mean velocity at the exit section of the actuator where $U$ and $p$ are the unknowns and $R e$ is the Reynolds number. The penalization term $U / K$ insures the velocity vanishes on the boundary of the solid parts $\bar{\Omega}_{S} \cap \bar{\Omega}_{F}$. The steady Stokes equations above are associated to the boundary conditions:

$$
\left\{\begin{array}{l}
U=(1,0) \quad \text { on } S_{\text {ent }}=S_{\text {ent }_{1}} \cup S_{\text {ent }_{2}} \cup S_{\text {ent }_{3}} \cup S_{\text {ent }_{4}} \\
U=(0,0) \text { on } \partial \Omega \cap \partial \Omega_{S} \\
\sigma(U, p) n=\sigma\left(U_{\text {ref }}, p_{\text {ref }}\right) n \text { on } S_{\text {exit }}
\end{array}\right.
$$

where the reference flow $\left(U_{r e f}, p_{r e f}\right)$ is defined either by the computed flow just before the exit section or by Poiseuille flow. These equations are solved using the finite differences multigrid method described in Subsection 2.3.

As the end of the actuator is located just at the end of the convergence of the four channels, the steady solution obtained exhibits there a profile somehow different to Poiseuille flow. So the method proposed in (Chantalat et al. 2009) is used to improve the design of the actuator and to get a profile superimposed to Poiseuille flow as we shall see at the end of this section.

\subsection{Optimization problem and definition of the adjoint equations}

In this section we show how to apply the method in the linear case. Our aim is to try to recover the Poiseuille 
flow, that is the asymptotic solution farther in the channel, right at the end of the actuator. To set the optimization problem, a cost function $J$ is defined according to the difference of the computed velocity and the target velocity (the Poiseuille flow) inside the strip $\Omega_{\text {strip }}$ at the beginning of the straight channel after the actuator:

$J\left(\Omega_{F}, U\left(\Omega_{F}\right)\right)=\frac{1}{2} \int_{\Omega_{\text {strip }}}\left|U-U_{\text {target }}\right|^{2} d \Omega$

where $U_{\text {target }}=\left(u_{1 \text { target }}, 0\right)=\left(u_{\text {Poiseuille }}, 0\right)$.

Then the adjoint problem is built in the fluid domain following the classical approach (Céa 1986; Guillaume and Sid 2004; Mohammadi and Pironneau 2010). Let us define the domains with respect to the level-set function $\phi$ in the following way. We set $\Omega_{F}=\{x / \phi(x)<$ $0\}, \Omega_{S}=\{x / \phi(x)>0\}$ and $\Gamma=\bar{\Omega}_{S} \cap \bar{\Omega}_{F}=$ $\{x / \phi(x)=0\}$. Then we introduce $\lambda: \mathbb{R}^{2} \rightarrow \mathbb{R}^{2}$, $\mu_{1}: \mathbb{R} \rightarrow \mathbb{R}$ and $\mu_{2}: \mathbb{R}^{2} \rightarrow \mathbb{R}^{2}$ three smooth enough real-valued functions referred as Lagrange multipliers. As presented in (Céa 1986), the minimization of the objective function $J$, subject to the direct Stokes problem, is equivalent to find the stationary points of the Lagrangian functional:

$$
\begin{aligned}
L((U, p), \phi)= & \frac{1}{2} \int_{\Omega_{\text {strip }}}\left|U-U_{\text {target }}\right|^{2} d \Omega \\
& +\int_{\{x / \phi(x)<0\}} \lambda \cdot\left(\frac{1}{\operatorname{Re}} \Delta U-\nabla p\right) d \Omega \\
& +\int_{\{x / \phi(x)<0\}} \mu_{1} \nabla \cdot U d \Omega \\
& +\int_{\{x / \phi(x)=0\}} \mu_{2} \cdot U d \Gamma .
\end{aligned}
$$

Let us suppose that $(\tilde{U}, \tilde{p})$ is an infinitesimal perturbation of the steady solution of the Stokes problem. Assuming that the perturbed flow satisfies $\tilde{U}=0$ on $\Gamma$ and $\tilde{U}=0$ on $S_{\text {ent }} \cup S_{\text {exit }}$, we can write using twice Green formula for the velocity perturbation:

$$
\begin{aligned}
& \delta L(\tilde{U}, \tilde{p})=\int_{\Omega_{\text {strip }}}\left(U-U_{\text {target }}\right) \cdot \tilde{U} d \Omega+\int_{S_{\text {ent }} \cup S_{\text {exit }} \cup\{x / \phi(x)=0\}} \lambda \cdot\left(\frac{1}{\operatorname{Re}} \nabla \tilde{U} n\right) d \Gamma \\
& -\int_{S_{e n t} \cup S_{e x i t} \cup\{x / \phi(x)=0\}} \frac{1}{R e}(\nabla \lambda n) \cdot \tilde{U} d \Gamma+\int_{\{x / \phi(x)<0\}} \frac{1}{R e} \Delta \lambda \cdot \tilde{U} d \Omega \\
& -\int_{S_{\text {ent }} \cup S_{\text {exit }} \cup\{x / \phi(x)=0\}}(\lambda \cdot n) \tilde{p} d \Gamma+\int_{\{x / \phi(x)<0\}}(\nabla \cdot \lambda) \tilde{p} d \Omega \\
& +\int_{S_{e n t} \cup S_{\text {exit }} \cup\{x / \phi(x)=0\}} \mu_{1}(\tilde{U} \cdot n) d \Gamma-\int_{\{x / \phi(x)<0\}} \nabla \mu_{1} \cdot \tilde{U} d \Omega \\
& +\int_{\{x / \phi(x)=0\}} \mu_{2} \cdot \tilde{U} d \Gamma \\
& \delta L(\tilde{U}, \tilde{p})=\int_{\{x / \phi(x)<0\}}\left(\frac{1}{R e} \Delta \lambda-\nabla \mu_{1}+\mathbb{1}_{\Omega_{\text {strip }}}\left(U-U_{\text {target }}\right)\right) \cdot \tilde{U} d \Omega+\int_{\{x / \phi(x)<0\}}(\nabla \cdot \lambda) \tilde{p} d \Omega \\
& +\int_{S_{e n t} \cup S_{e x i t} \cup\{x / \phi(x)=0\}} \lambda \cdot\left(\frac{1}{R e} \nabla \tilde{U} n\right) d \Gamma-\int_{S_{e n t} \cup S_{e x i t} \cup\{x / \phi(x)=0\}}(\lambda \cdot n) \tilde{p} d \Gamma \\
& +\int_{\{x / \phi(x)=0\}}\left(\mu_{1} n-\frac{1}{R e} \nabla \lambda n+\mu_{2}\right) \cdot \tilde{U} d \Gamma
\end{aligned}
$$

where $\mathbb{1}_{\Omega}$ is the characteristic function of $\Omega$ and $n$ is the unit normal vector on the fluid boundary pointing outside of the fluid zone.

Hence, the adjoint problem satisfied by $\left(\lambda, \mu_{1}\right)$ is:

$$
\left\{\begin{array}{l}
-\frac{1}{R e} \Delta \lambda+\nabla \mu_{1}=\mathbb{1}_{\Omega_{\text {strip }}}\left(U-U_{\text {target }}\right) \text { in } \Omega_{F} \\
\nabla \cdot \lambda=0 \text { in } \Omega_{F} \\
\lambda=0 \text { on } \Gamma \cup S_{\text {ent }} \cup S_{\text {exit }} .
\end{array}\right.
$$

This problem is solved using the volume penalization method like the direct problem:

$$
\left\{\begin{array}{l}
-\frac{1}{R e} \Delta \lambda+\nabla \mu_{1}+\frac{\lambda}{K}=\mathbb{1}_{\Omega_{\text {strip }}}\left(U-U_{\text {target }}\right) \text { in } \Omega \\
\nabla \cdot \lambda=0 \text { in } \Omega \\
\lambda=0 \text { on } S_{\text {ent }} \cup S_{\text {exit }} .
\end{array}\right.
$$


Once (13) are solved by the same method used for the direct problem, the Lagrange multiplier $\mu_{2}$ on the interface fluid/solid is given by:

$\mu_{2}=\frac{1}{R e} \nabla \lambda n-\mu_{1} n$ on $\Gamma$.

Then a perturbation of the Lagrangian functional $L$ with respect to a small modification of the level-set function $\tilde{\phi}$ is computed as:

$$
\begin{aligned}
\delta L(\tilde{\phi})= & \delta J(\tilde{\phi})-\int_{\{x / \phi(x)=0\}} \partial_{n}\left(\mu_{2} \cdot U\right) \tilde{\phi} d \Gamma \\
= & -\frac{1}{2} \int_{\partial \Omega_{\text {strip }}}\left|U-U_{\text {target }}\right|^{2} \tilde{\phi} d \Gamma \\
& -\int_{\{x / \phi(x)=0\}}\left(\frac{1}{2} \partial_{n} \lambda-\mu_{1} n\right) \cdot \partial_{n} U \tilde{\phi} d \Gamma .
\end{aligned}
$$

Thus, as we impose $\tilde{\phi}=0$ in the straight channel beyond the actuator and on the other hand $n \cdot \partial_{n} U=0$ on $\Gamma$, the modification of the solid domain due to the optimization process is given by:

$\tilde{\phi}=\partial_{n} U \cdot \partial_{n} \lambda$ on $\Gamma$,

which is equivalent to transport the level-set function at the boundary (as in (4)) in the normal direction with the speed $\tilde{\phi}$ when $\phi$ is a distance function. Finally, to take into account the constraints on the domain (fixed entrance and exit sections of the actuator), the modification of the domain are rewritten on the whole domain $\Omega$ as:

$\tilde{\phi}=\mathbb{1}_{\text {actuator }} \partial_{n} U \cdot \partial_{n} \lambda$ in $\Omega$

where $\mathbb{1}_{\text {actuator }}$ is the characteristic function of the first half open part of the domain between the entrance and exit sections of the actuator.

\subsection{Results for the Stokes flow}

In this subsection, the level-set adjoint formulations above are used in order to optimize the actuator so that the profile of the flow at the exit section of the actuator is a Poiseuille profile. There is a strong constraint as the four entrance sections and the exit section cannot be changed. Thus the optimization method can only improve the inside part of the actuator geometry (including entrance obstacles) up to the exit channel beginning.

In Fig. 4 the convergence of the cost function $J$ (see (18)) versus the number iterations is plotted. As the figure shows the optimization convergence is almost achieved after about 80 iterations. The functional is minimized after 80 iterations and during all the procedure even if it slightly oscillates with some higher values, no significant increase is observed. In this respect it should be noticed that we use a gradient method without line search. This means that a local increase of the functional may occur.

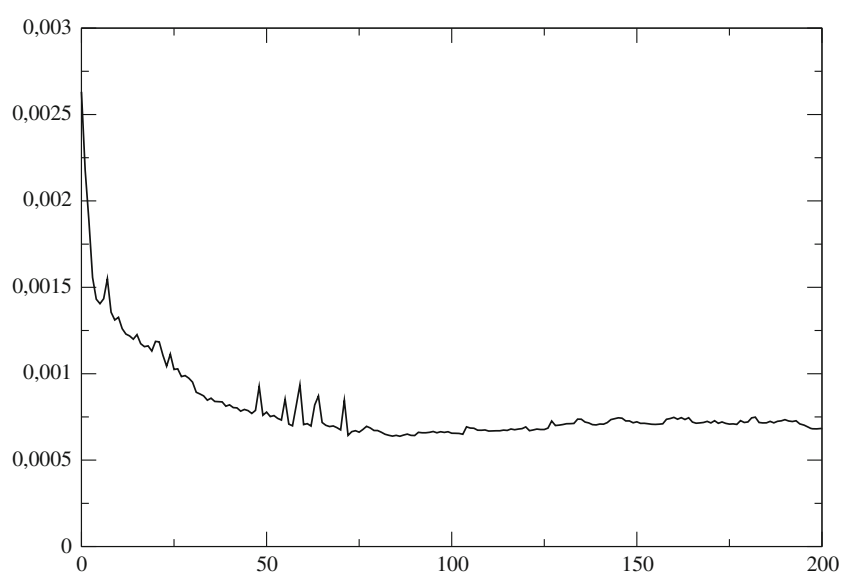

Fig. 4 Evolution of the cost function $J$ during the shape optimization iterations

Figure 5 shows the velocity profiles at the exit section of the actuator. There is a comparison between the initial profile obtained with the direct numerical simulation on the initial geometry and the profile obtained after the optimization process. A first remark is that for Stokes flow the initial profile is close to Poiseuille flow contrarily to the profile obtained with Navier-Stokes solver (see Fig. 3). After the shape optimization in order to minimize $J$ is achieved the velocity profile is superimposed to Poiseuille profile. There is no noticeable difference between the optimized and the target results. Thus the shape optimization process is validated as the target is reached. The initial actuator geometry is compared to the optimized one in Fig. 6. The geometry is unchanged in the main parts of the actuator except the sharp edges of the solid parts between the entrance channels that have been smoothed and the end of the convergent part that has been contracted to slow down the flow. These

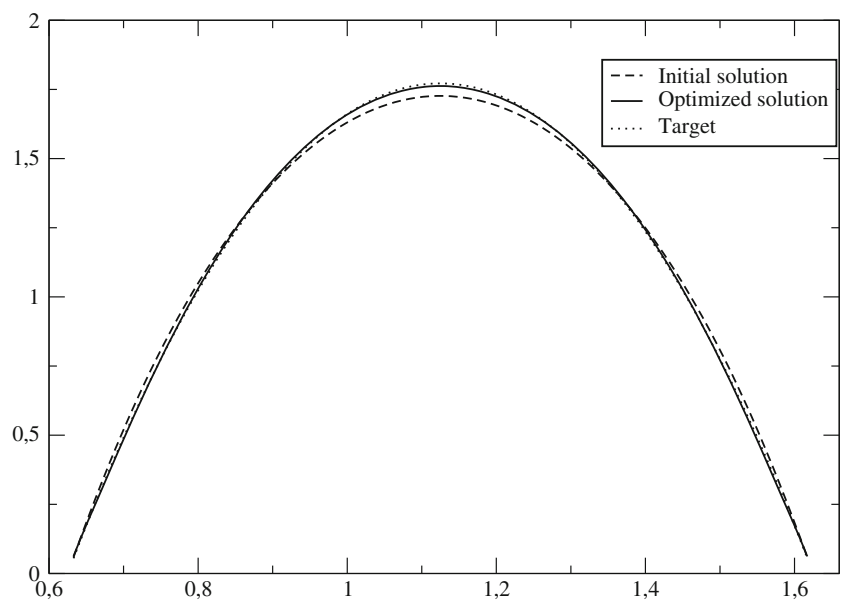

Fig. 5 Profiles of the velocity at the exit section of the actuator 


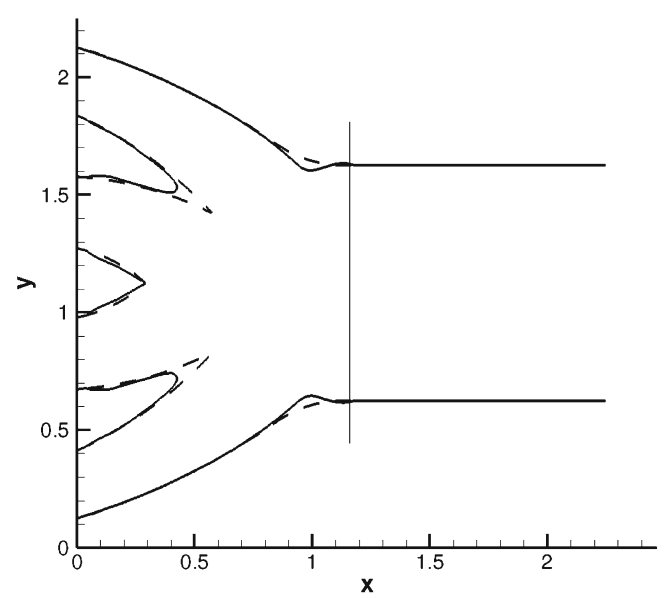

Fig. 6 Actuator geometry: initial geometry in dashed lines and final geometry in solid lines. The vertical line corresponds to the exit section of the actuator

modifications decrease the jet effects to recover the Poiseuille profile.

\section{Shape optimization for a Navier-Stokes flow}

In the previous section the optimization process has been described for the linear Stokes problem. Here we show how to adapt the method to the nonlinear Navier-Stokes equations.

\subsection{Optimization process}

The main difficulty is linked to the fact that the stable Navier-Stokes solution is not steady any more and so it is not possible to compare the solution to a target as there is not one solution but a set of solutions depending on the time. One possibility is to compare a mean solution to a target. However, on the one hand it is not clear how to define the mean solution except for a purely periodic flow choosing a time period and on the other hand a mean solution is not an instantaneous solution. Consequently the optimization process does not make sense. Besides, we want to avoid a time-dependent optimization problem because it would lead to an expensive computational set up. So we decide to overcome that difficulty using the unstable steady solution $\left(U_{S}, p_{S}\right)$ as it is one mathematical solution that is directly linked to the geometry. As this solution is unstable, a continuation method could be necessary to capture it but we use a trick to avoid extra CPU time. Indeed, to get that solution, the initial solution of the direct problem is set to $(U, p)=(0,0)$ and the iteration process drives first the iterates to the unstable steady solution before moving to the stable evolution solution. So it suffices to stop when the steady solution is reached. Thus, for the optimization we can keep the same cost function $J$ inside the strip $\Omega_{\text {strip }}$ than for the linear case:

$J\left(\Omega_{F}, U_{S}\left(\Omega_{F}\right)\right)=\frac{1}{2} \int_{\Omega_{\text {strip }}}\left|U_{S}-U_{\text {target }}\right|^{2} d \Omega$

where $U_{\text {target }}=\left(u_{1 \text { constant }}, 0\right)$. That target is a little bit surprising as a constant of course is not a solution but for the car industry it is necessary to get a flow at the end of the actuator as constant as possible.

Here, at each optimization step, replacing Stokes equations by steady Navier-Stokes equations as the perturbation occurs in the vicinity of the unstable steady solution, the Lagrangian functional becomes (Mohammadi and Pironneau 2010; Zhou and Li 2008):

$$
\begin{aligned}
L\left(\left(U_{S}, p_{S}\right), \phi\right)= & \frac{1}{2} \int_{\Omega_{\text {strip }}}\left|U_{S}-U_{\text {target }}\right|^{2} d \Omega \\
& +\int_{\{x / \phi(x)<0\}} \lambda \cdot\left(\frac{1}{R e} \Delta U_{S}-\nabla p_{S}-\left(U_{S} \cdot \nabla\right) U_{S}\right) d \Omega \\
& +\int_{\{x / \phi(x)<0\}} \mu_{1} \nabla \cdot U_{S} d \Omega \\
& +\int_{\{x / \phi(x)=0\}} \mu_{2} \cdot U_{S} d \Gamma
\end{aligned}
$$

The only difference with the linear case is due to the convection term. Let us set:

$L_{c o n v}\left(\left(U_{S}, p_{S}\right), \phi\right)=\int_{\{x / \phi(x)<0\}} \lambda \cdot\left(-\left(U_{S} \cdot \nabla\right) U_{S}\right) d \Omega$,

it comes for an infinitesimal perturbation $(\tilde{U}, \tilde{p})$ of the steady solution:

$$
\begin{array}{r}
\delta L_{\text {conv }}(\tilde{U}, \tilde{p})=\int_{\{x / \phi(x)<0\}} \lambda \cdot(-( \\
\left.\left(U_{S}+\tilde{U}\right) \cdot \nabla\right) \\
\left.\left(U_{S}+\tilde{U}\right)\right) d \Omega .
\end{array}
$$

The quadratic term $\left(U_{S} \cdot \nabla\right) U_{S}$, associated to the other terms vanishes. So, if the quadratic term $(\tilde{U} \cdot \nabla) \tilde{U}$ is neglected we get:

$$
\begin{aligned}
\delta L_{\text {conv }}(\tilde{U}, \tilde{p}) \simeq & \int_{\{x / \phi(x)<0\}} \lambda \cdot\left(-\left(U_{S} \cdot \nabla\right) \tilde{U}\right) d \Omega \\
& +\int_{\{x / \phi(x)<0\}} \lambda \cdot\left(-(\tilde{U} \cdot \nabla) U_{S}\right) d \Omega .
\end{aligned}
$$

Integrating the first term by part, as $U_{S}$ is divergence free:

$$
\begin{aligned}
\delta L_{\text {conv }}(\tilde{U}, \tilde{p}) \simeq & \int_{\{x / \phi(x)<0\}}\left(\left(U_{S} \cdot \nabla\right) \lambda\right) \cdot \tilde{U} d \Omega \\
& -\int_{\{x / \phi(x)=0\}}\left(U_{S} \cdot n\right) \lambda \cdot \tilde{U} d \Gamma \\
& +\int_{\{x / \phi(x)<0\}}\left(-\left(\nabla U_{S}\right)^{t} \lambda\right) \cdot \tilde{U} d \Omega .
\end{aligned}
$$


Thus the adjoint problem becomes similarly to Stokes case (12):

$$
\left\{\begin{array}{l}
-\frac{1}{R e} \Delta \lambda+\nabla \mu_{1}=\mathbb{1}_{\Omega_{\text {strip }}}\left(U_{S}-U_{\text {target }}\right) \\
+\left(U_{S} \cdot \nabla\right) \lambda-\left(\nabla U_{S}\right)^{t} \lambda \text { in } \Omega_{F} \\
\nabla \cdot \lambda=0 \text { in } \Omega_{F} \\
\lambda=0 \text { on } \Gamma \cup S_{\text {ent }} \cup S_{\text {exit }} .
\end{array}\right.
$$

Starting with the initial domain, the level-set function $\phi$ is defined and the time-dependent Navier-Stokes equations are solved to get the unstable steady solution $U_{S}$. Then the adjoint problem is solved using the volume penalization method as previously to get $\lambda$ and the perturbation of the domain is given like in the linear case by:

$\tilde{\phi}=\mathbb{1}_{\text {actuator }} \partial_{n} U_{S} \cdot \partial_{n} \lambda$ in $\Omega$.

\subsection{Results for Navier-Stokes flow}

During the optimization process we compute a sequence of unstable steady solutions $\left(U_{S}\right)_{p}$ for $p$ varying from 1 to the number of changes of the domain shape. This number is achieved when $\tilde{\phi}$ is small enough, that is when the relative Euclidean norm of $\left(U_{S}\right)_{p}$ is small enough or the cost function $J\left(\Omega_{F}, U_{S}\left(\Omega_{F}\right)\right)$ does not change much from one step to another. Numerically, it is not so easy to get the unstable steady solution as we have to stop the computation when the time solution starts to be carried away to the stable unsteady solution. The test on the steadiness is not very accurate and so the detected steady solution can change too much from one step to another. To smooth the optimization process the unstable steady solution $\left(U_{S}\right)_{p}$ is defined as the mean of four consecutive solutions. Then the convergence is achieved as shown in the Fig. 7 where the history of the cost function is plotted. Like in Stokes case a local increase of the functional can occur. The iteration process is much

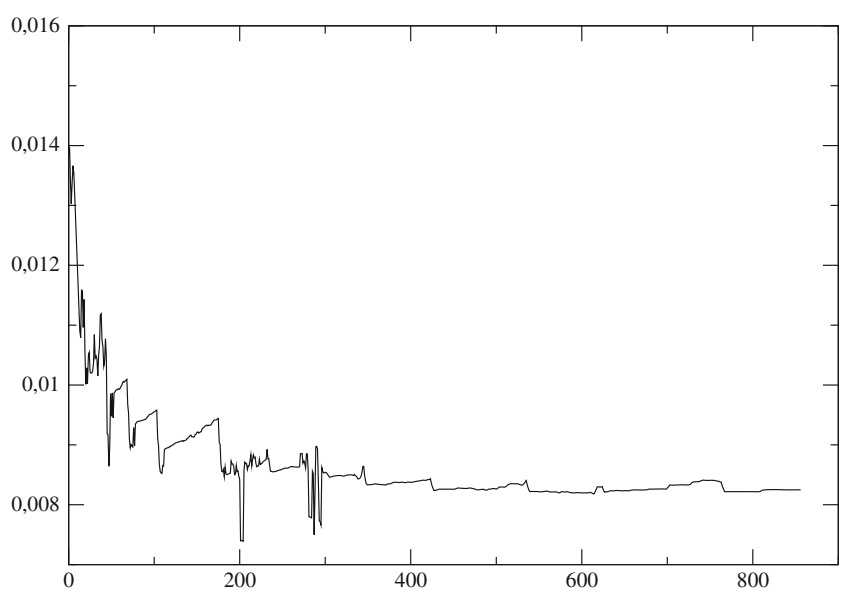

Fig. 7 Evolution of the cost function $J$ during the shape optimization iterations

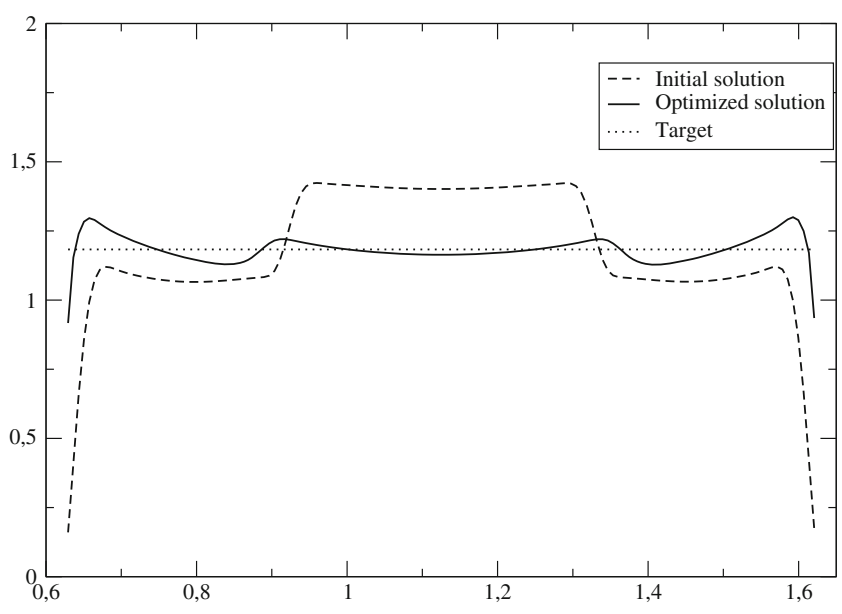

Fig. 8 Profiles of the steady velocity at the exit section of the actuator

longer than in the linear case but a good convergence is achieved. The first indicator to judge the efficiency of the method is to compare the steady solution $U_{S}$ to the target $U_{\text {target }}$ which is a constant at the exit section of the actuator. The velocity profiles are plotted in Fig. 8. One can see that, even if a constant is not a solution, the profile of the steady solution at convergence is much closer to the constant than $\left(U_{S}\right)_{1}$ obtained on the initial domain. The main difference is due to the no-slip boundary condition on the walls of the channel.

A question is how does the mean profile of timedependent Navier-Stokes solution behave? We have seen in Subsection 2.3 that this mean profile exhibits four peaks corresponding to the four channels. We can see in Fig. 9 that the mean profile has changed drastically as there is no more peaks in the middle. Nevertheless two peaks are still present in the vicinity of the walls of the channel.

Let us see now what is the final shape of the actuator which is plotted in Fig. 10. The solid parts between the

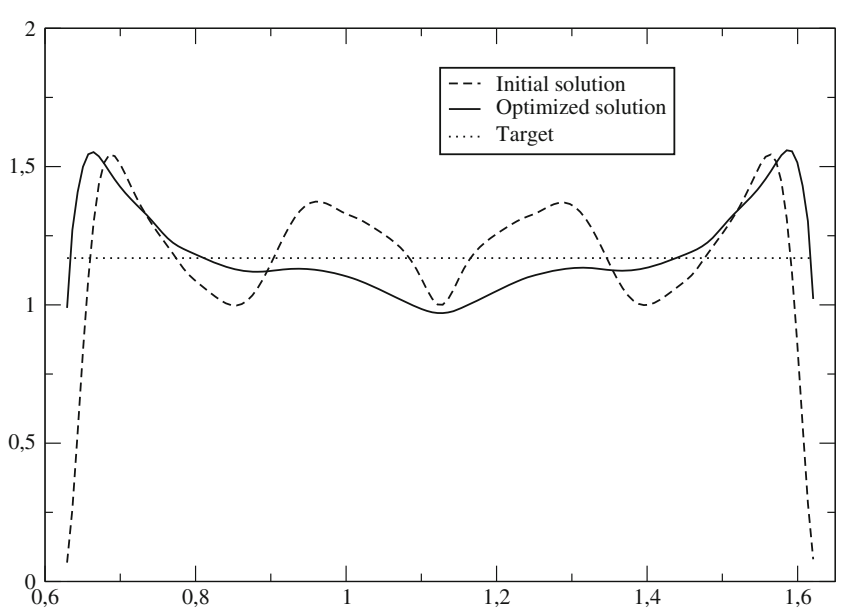

Fig. 9 Profiles of the mean velocity at the exit section of the actuator 


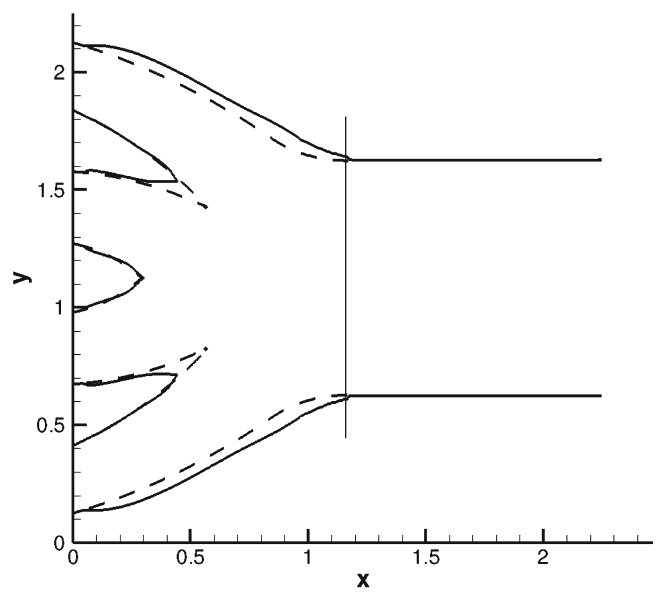

Fig. 10 Actuator geometry: initial geometry in dashed lines and final geometry in solid lines. The vertical line corresponds to the exit section of the actuator

four entrance channels are modified about the same way than in the linear Stokes case. The main difference concerns the walls of the channel that are enlarged. These modifications decrease the jet effect of the four channels as the fluid domain is more open. The result is quite satisfactory even if the target is not fully reached. To apply the result in the industry it is necessary to perform a polynomial approximation of each side of the four channels without changing the entrance and exit points. This is achieved for instance by classical polynomial interpolation or using Bezier curves. The value of the cost function does not change after such a modification as the final shape of the channels is regular and can be approximated as accurately as needed.

\section{Conclusions}

A shape optimization method is applied to improve the shape of an active flow control actuator in order that the velocity profile at the exit section tends to a given target profile. The proposed algorithm is based on the penalization and the level-set methods with Cartesian grids to handle the solid parts and follow the fluid-solid interfaces. Both the use of Cartesian grids and of steady solutions in the nonlinear case make the optimization method very efficient in terms of cpu time. In the Stokes linear case the method improves the geometry so efficiently that the velocity profile is superimposed to the target profile. The extension to the Navier-Stokes nonlinear case yields good results even if the target is not completely reached. It is noticeable that the method is able to change globally the shape of the actuator without using several parameters.

Acknowledgments This work was carried out in the framework of the CARAVAJE project supported by the Agence pour le
Développement Et la Maîtrise de l'Energie (ADEME). We thank FLOWDIT research team for the initial geometry of the actuator and all the partners for fruitful discussions.

\section{References}

Allaire G, Jouve F (2006) Coupling the level set method and the topological gradient in structural optimization. In: Bendsoe $M$ et al (ed) IUTAM symposium on topological design optimization of structures, machines and materials. Springer-Verlag, Heidelberg

Amstutz S (2005) The topological asymptotic for the Navier-Stokes equations. ESAIM Control Optim Calc Var 11( $\left.n^{\circ} 3\right)$

Angot Ph, Bruneau Ch-H, Fabrie P (1999) A penalization method to take into account obstacles in incompressible viscous flows. Numer Math 81

Bendsøe MP, Sigmund O (2003) Topology optimization: theory, methods and applications. Springer-Verlag, Heidelberg

Borrvall T, Petersson J (2003) Topology optimization of fluids in Stokes flow. Internat J Numer Methods Fluids $41\left(n^{\circ} 1\right)$

Brackett DJ, Ashcroft IA, Hague RJ (2010) Multi-physics optimisation of 'brass' instruments-a new method to include structural and acoustical interactions. Struct Multidiscip Optim 40

Bruneau Ch-H, Mortazavi I (2008) Numerical modelling and passive flow control using porous media. Comput \& Fluids $37\left(n^{\circ} 5\right)$

Bruneau C-H, Saad M (2006) The 2D-lid driven cavity problem revisited. Comput \& Fluids $35\left(n^{\circ} 3\right)$

Céa J (1986) Conception optimale ou identification de formes: calcul rapide de la dérivée directionnelle de la fonction coût. Math Model Numer Anal 20( $\left.n^{\circ} 3\right)$

Chantalat F (2009) Méthode level-set et de pénalisation pour l'optimisation et le contrôle d'écoulements Ph D Thesis University Bordeaux 1

Chantalat F, Bruneau C-H, Galusinski C, Iollo A (2009) Levelset, penalization and meshes cartesian: a paradigm for inverse problems and optimal design. J Comput Phys 228( $\left.n^{\circ} 17\right)$

Duan X, Ma Y, Zhang R (2008) Optimal shape control of fluid flow using variational level set method. Phys Lett A 372( $\left.n^{\circ} 9\right)$

Gad-el-Hak M (1999) The fluid mechanics of microdevices-the freeman scholar lecture. J Fluids Eng 121

Gersborg-Hansen A, Sigmund O, Haber RB (2005) Topology optimization of channel flow problems. Struct Multidiscip Optim 30

Guillaume Ph, Sid Idris K (2004) Topological sensitivity shape optimization for the Stokes equations. SIAM J Control Optim 43( $\left.n^{\circ} 1\right)$

Jiang GS, Shu CW (1996) Efficient implementation of weighted ENO schemes. J Comput Phys $126\left(n^{\circ} 1\right)$

Mohammadi B, Pironneau O (2010) Applied shape optimization for fluids, 2nd edn. Numerical mathematics and scientific computation. Oxford University Press, London

Osher S, Fedkiw R (2001) Level set methods: an overview and some recent results. J Comput Phys 169( $\left.n^{\circ} 2\right)$

Osher S, Santosa F (2001) Level set methods for optimization problems involving geometry and constraints. I. Frequencies of a two-density inhomogeneous drum. J Comput Phys $171\left(n^{\circ} 1\right)$

Osher S, Sethian JA (1988) Fronts propagating with curvaturedependent speed: algorithms based on Hamilton-Jacobi formulations. J Comput Phys 79( $\left.n^{\circ} 1\right)$

Sethian JA (1996) A fast marching level set method for monotonically advancing fronts. Proc Natl Acad Sci USA 93

Sethian JA (2001) Evolution and application of level-set and fastmarching methods for advancing fronts. J Comput Phys $169\left(n^{\circ} 2\right)$

Zhou S, Li Q (2008) A variational level set method for the topology optimization of steady-state Navier-Stokes flow. J Comput Phys $227\left(n^{\circ} 24\right)$ 